\title{
A Unified Authentication Scheme Based on Analytic Mechanism
}

\author{
Yong $\mathrm{Xu}$, Yejuan Cheng and Chang Xiao \\ School of Economics and Commerce, South China University of Technology, \\ Guangzhou 510006, China \\ xuyong@scut.edu.cn,cyj19910813@163.com,xiaochangwy163@163.com
}

\begin{abstract}
To resolve security issues and management issues of different Certificate Authorities $(C A s)$, this paper proposed a unified authentication scheme, which will make different Certificate Authorities (CAs) be uniformly authenticated. In addition, it showed the whole structure of the scheme, added an extensional registration number to certificates and designed unified authentication process for the scheme. The unified authentication process was modelled and simulated by Coloured Petri Net (CPN) Tools and verified by analyzing the state space of CPN model. In conclusion, this paper provides a unified authentication service and confirms that the proposed scheme is totally controllable, realizable and feasible. The suggested scheme can enhance the efficiency and security of authentication systems and achieve good results in practical applications.
\end{abstract}

Keywords: unified authentication scheme, certificate authority (CA), Coloured Petri Net

\section{Introduction}

With the rapid development of e-commerce and online payment systems, a great deal of certificate authority (CA) systems have been built [1-2]. But due to multiple Root CAs and compatibility issues of different CAs, it is difficult for these systems to achieve unified scheduling and unified management, which has great effect on payment security.

To achieve a unified authentication between CAs, the literature [3] mentioned crosscertification. Casola indicated that cross certification among CAs is a very huge problem for security experts whom trying to understand if two CAs could cooperate with each other, hence, he proposed a methodology to automatically evaluate and compare security policies for Cross Certification [4]. Turnbull explained two methods to extend trust between certification authorities (CAs): peer-to-peer cross-certification and hierarchical crosscertification [5]. He Yu-Jin has structured a unified cross-certification center based on PKI [6].

Except for cross-certified meshes, Linn has defined several other approaches to link certification structures, including subordinated hierarchies, hybrids and bridge CAs [7]. Alterman proposed the US Federal PKI, which is designed around the Federal Bridge Certification Authority [8]. The Bridge Certification Authority (CA) provides a new way to link the capabilities of existing corporate PKIs [9].With the emergence of cloud computing, CA technology has undergone a renaissance, enabling different computers to communicate directly [10-12].

In this paper, we design a unified authentication scheme for CAs based on analytic mechanism, the scheme we proposed is able to make all CAs be authenticated mutually. Our contributions are mainly in two folds. First of all, we propose a different unified authentication process. Secondly, we model the process by CPN tools and verify it. 
The rest of this paper is organized as follows. In the next section, we give a brief overview about the scheme. Then, we describe the unified authentication scheme in Section 3 and model its process by CPN tools in Section 4. In Section 5, we verify the process by analyzing the state space of CPN model. Finally, we conclude our paper with future research directions in Section 6.

\section{Preliminaries}

The structure of unified authentication scheme is composed by analytic center of top $\mathrm{CA}$, analytic center of district CA, root CAs, subordinate CAs and users, which is shown in Figure 1.

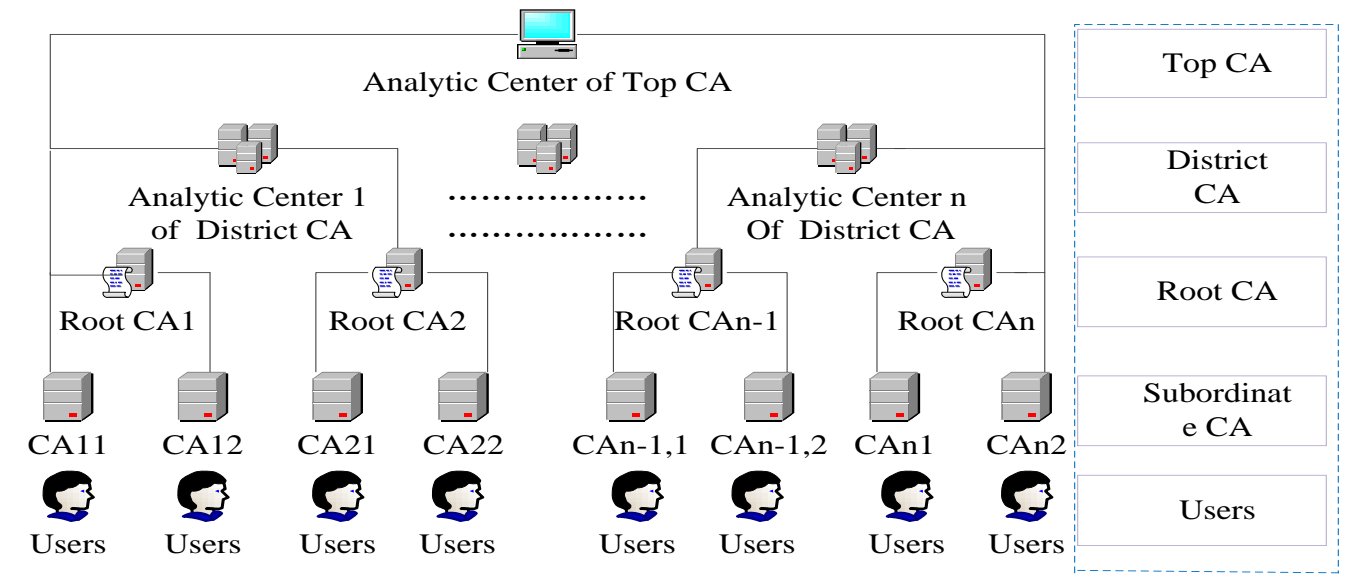

Figure 1. Framework of the Scheme

Differing from traditional CA systems, the unified authentication mechanism mostly depends on analytic center of CA, which not only links root CAs, subordinated CAs and users in a same district, but also achieves authentication in different districts. Meanwhile, a digital certificate in unified authentication scheme integrates different types of existing certificates, so users can pay in different payment platforms by one certificate. Besides, analytic centers of CA provide security services for users to ensure the safe authentication.

\section{The Unified Authentication Scheme}

\subsection{Registration Process for Analytic Centers of CA}

In a unified authentication scheme, there are ten steps for root CA to become authenticated user through analytic centers of CA, as shown in Figure 2. Firstly, the root $\mathrm{CA}$ submits an application and related materials to the district $\mathrm{CA}$, then pending review. After the application is confirmed by analytic center of district $\mathrm{CA}$, the center will submit the application to analytic center of top CA, then pending review. When analytic center of top CA confirmed the application, a unique user's identifier will be generated and user's data will be imported into users' directories. Meanwhile, the approved information of user's application and the identity of successful registration are returned by analytic center of top CA. Then analytic center of district CA will import user's data into users' directories and identify the registration, besides, the 
center will store the unified digital certificate. Finally, with the confirmation message returned to analytic center of district $\mathrm{CA}$, the root $\mathrm{CA}$ is registered successfully.

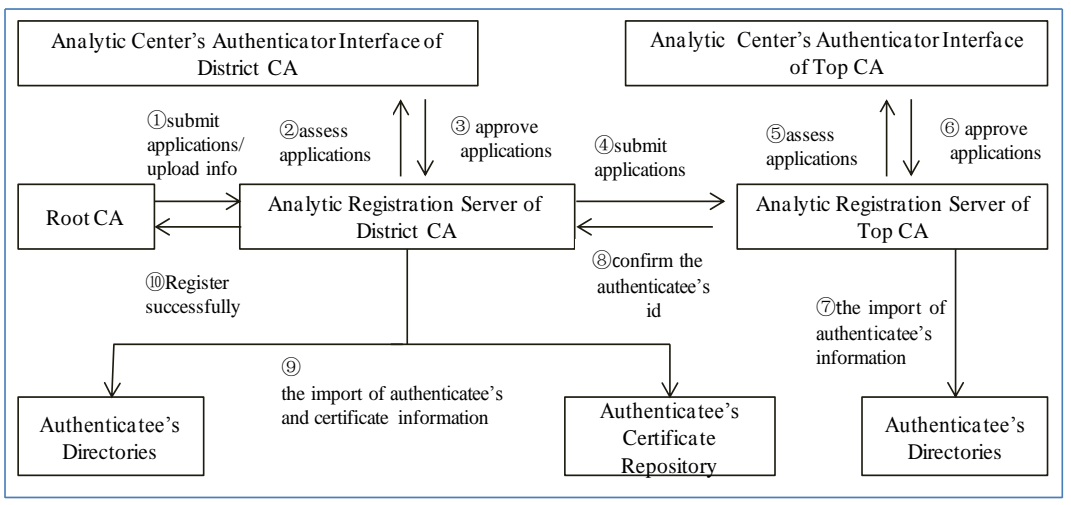

Figure 2. Registration Process

\subsection{Digital Certificate based on Unified Authentication}

Digital certificate is a digitally signed statement, including version information about the certificate, serial number of the certificate, signature algorithm, the name of issuer, validity period, the owner of the certificate, public keys and the certificate signature [13-14].There are three versions about PKI digital certificates, including X.509.v1, X.509.v2, X.509.v3, and the X.509.v3 is the most popular digital certificate [15-16].

In the unified authentication mechanism, CA analytic centers will generate a registration number for each root $\mathrm{CA}$, meantime, each root $\mathrm{CA}$ will create registration numbers for subordinate CAs or users when digital certificates are issued.

Certificate owner can be positioned by extensional registration number, so CA analytic centers and different CAs at all levels could build a trust chain by using the number. Data constraint of extensional registration number is shown in Table 1.

Table 1. Data Constraints of Extensional Registration Number

\begin{tabular}{|c|c|c|}
\hline Name & Format and Constraint & Description \\
\hline $\begin{array}{c}\text { Registration } \\
\text { Number }\end{array}$ & String & $\begin{array}{c}\text { Location and identification of digital } \\
\text { certificates' entity }\end{array}$ \\
\hline
\end{tabular}

In this paper, to achieve unified authentication both in a same district and different districts, the Registration Number which can illustrate the location of certificate's owner is added in extended domain of X.509 V3 certificates. The format of digital certificate in the unified authentication scheme is shown in Table 2.

Table 2. Data Definition of Unified Authentication's Digital Certificate

\begin{tabular}{|c|c|c|}
\hline Name & $\begin{array}{c}\text { Format and } \\
\text { Constraints }\end{array}$ & Description \\
\hline Version & Integer & Certificate version, always set to 3. \\
\hline Issuer Name & Name & Distinguished Name(DN) of CA. \\
\hline Validity. not Before & UTC time & The time specifying the certificate is valid. \\
\hline Validity. not After & UTC time & The certificate's expiration time. \\
\hline Subject Name & Name & DN for an entity who has a key. \\
\hline
\end{tabular}




\begin{tabular}{|c|c|c|}
\hline Serial Number & Integer & The serial number specified by the CA. \\
\hline Algorithm Identifier & OID and type & $\begin{array}{c}\text { Including address code, identification code } \\
\text { and serial number. }\end{array}$ \\
\hline $\begin{array}{c}\text { Subject Public Key Info. } \\
\text { Algorithm. Algorithm } \\
\text { Identifier }\end{array}$ & OID and type & The algorithm can be used to specify the key. \\
\hline $\begin{array}{c}\text { Subject Public key Info } \\
\text {.Subject Public key }\end{array}$ & Bit string & The shared key in the certificate request. \\
\hline $\begin{array}{c}\text { Issuer's Unique } \\
\text { Identifier }\end{array}$ & Bit & $\begin{array}{c}\text { When a same name of X.500D is used for } \\
\text { multiple certification issuers, using a bit of } \\
\text { string to identify name of certificate holders. }\end{array}$ \\
\hline $\begin{array}{c}\text { User's Unique Identifier } \\
\text { mhen a same name of X.500D is used for } \\
\text { multiple certification holders, using a bit of } \\
\text { string to identify name of certificate holders. }\end{array}$ \\
\hline Signature Value & String & $\begin{array}{c}\text { The value of certificate issuing authority's } \\
\text { certificate signature. }\end{array}$ \\
\hline Extensions. extn Value & STRING & $\begin{array}{c}\text { Location of digital certificate's subject and } \\
\text { the identity issued by certificate authorities. }\end{array}$ \\
\hline
\end{tabular}

In accordance with the certificate format mentioned above, we identify Cert (certificates in a unified authentication scheme) as a seven-tuples, C = Cert (I, DS, DE, S, PK, E, Sig). And related certificates' functions are shown in Table 3 and Table 4.

Table 3. Certificate Items and Tuple Symbol

\begin{tabular}{|c|c|c|c|}
\hline Certificate Item & Symbol & Certificate Item & Symbol \\
\hline issuer of cert & I & public key value & PK \\
\hline date of issue & DS & extension & E \\
\hline date of expiration & DE & value of signature & Sig \\
\hline subject of cert & S & & \\
\hline
\end{tabular}

Table 4. Related Certificates' Functions

\begin{tabular}{|c|c|}
\hline Demand & Function \\
\hline Solving the certificate issuer & GetI (C)=I \\
\hline Solving the date of issue & GetDS (C)=DS \\
\hline Solving the date of expiration & GetDE (C)=DE \\
\hline Solving the certificate extension & GetE $($ C) $=$ E \\
\hline The current date & D (Current)=Current \\
\hline
\end{tabular}

\subsection{Message Protocol based on Unified Authentication}

Message is data units switched and transferred in the network, as well as a data packet to be sent by site. And message contains complete information of data packet to be sent, its length is inconsistent and variable. In our unified authentication scheme, there are abundant digital transmissions between CAs and analytic center of CAs. To improve the efficiency of data transmission and authentication, we develop a unified authentication message protocol, which is based on the original message and integrates transmission information from different unified authentication mechanism parties.

Unified authentication message is composed of three parts, identifiers of message type, bit codes and the field value.

- Message type identifier: It is a 4-digit numeric field, which describes category and function of each message: 
0001: Authorization Message

0010: Check Message

0100: Successful Authentication Message

1000: Unsuccessful Authentication Message

- Bit code table: It is a bit map, constituted by 30-bit codes, '0' or ' 1 'represents contents of the corresponding message field from V1 to V30 is empty or not, where ' 1 ' means not empty and ' 0 ' means empty.

- Field value: It is consisted of a series of field value, these field values are arranged by the unified authentication message's provision, and each field value corresponds to a specific message.

\subsection{The Unified Authentication Process}

The proposed process has two aspects, including unified authentication within a district and unified authentication between different districts.

3.4.1. Authentication Process within a District: In the unified authentication process within a district, unified authentication digital certificates are just authorized by analytic centers of district CA, without top CA involved in the authentication process. For example, Bob need to verify the public key of Alice's certificate, as the same district's users, the whole unified authentication process is shown in Figure 3.

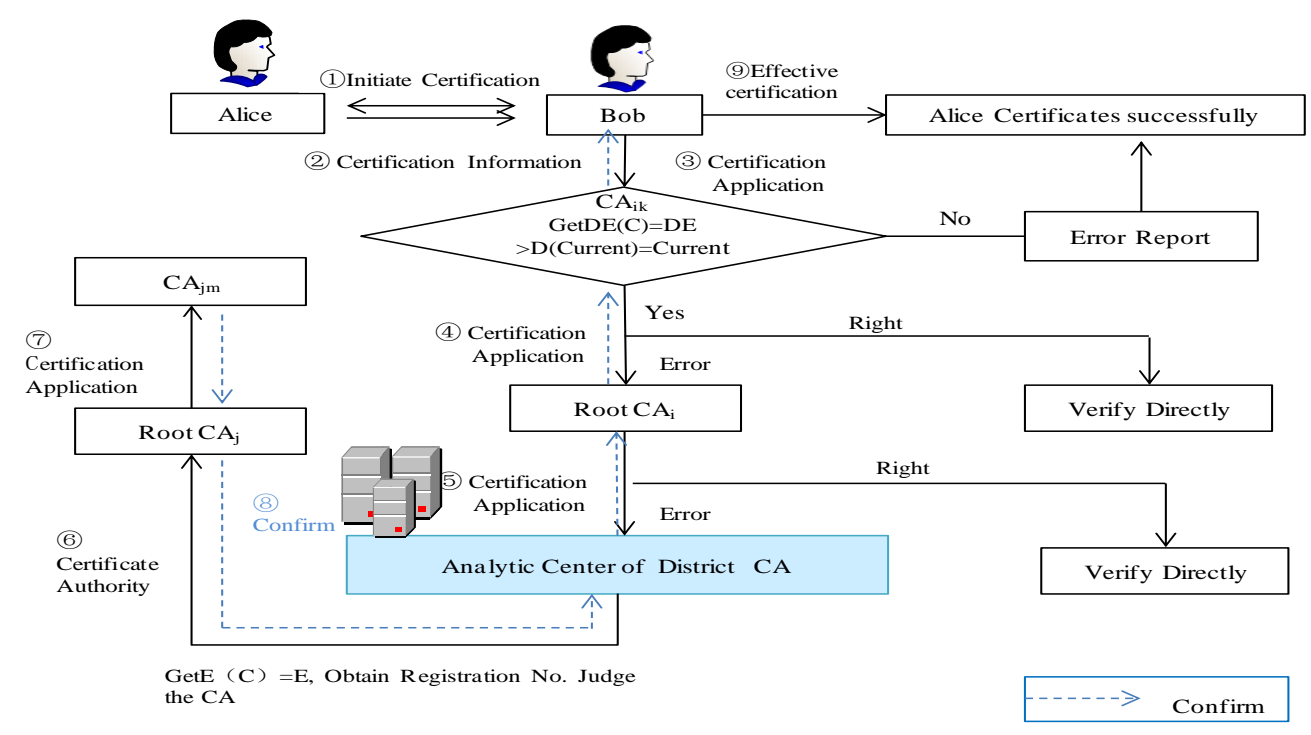

Figure 3. Flow Chart of the Authentication Process within a District

There are nine steps for the process within a district.

(1) Bob needs to verify the authenticity of the public key from Alice's certificate, so they launch authentication to each other.

(2) Bob gets Alice's certificate information.

(3) Bob issues a certification application to his own subordinate $C A_{i k}$.

(4) With information passed by Bob, $C A_{i k}$ judges validity of the certificate. If GetDE $(C)=D E>D$ (Current) $=$ Current, the certificate has not expired. Then $C A_{i k}$ 
continue to determine whether the certificate issued by its own CA, and if so, $C A_{i k}$ will verify directly, if it is not, $C A_{i k}$ will submit application to the root CA. if GetDE $(C)=D E<D$ (Current) = Current, where means that the certificate has expired, then an error report will be returned to the applicant, Alice.

(5) To determine whether the certificate issued by its own subordinate CA, Root $C A_{j}$ will inspect certificate information. If it is not the correct subordinate CA, Root $C A_{i}$ will apply for authentication from analytic center of district CA.

(6) Analytic center of district $\mathrm{CA}$ tests information of digital certificates and obtains a registration number $\left(G\right.$ et $E(C)=E$ ), then it authorizes the $\operatorname{root} C A_{j}$.

(7) Root $C A_{j}$ inspects certificate information, applying for certificate from $C A_{j m}$.

(8) $C A_{j m}$ inspects certificate information to determine whether the certificate is a valid certificate issued by its own CA. If so, the certificate is confirmed, and confirmation is backtracked. If it is not, the certificate is determined to be invalid, a confirmation message is backtracked.

(9) Bob receives the confirmation message, then he judges the validity of confirmation from certificate, finally, Alice will be confirmed if the certificate is valid.

3.4.2. Authentication Process between Different Districts: Differing from the unified authentication within a district, the unified authentication between different districts is the certificate authorization for users from different districts, and top CA is involved in this process. The whole unified authentication process is shown in Figure 4 (Bob and Tom come from different districts).

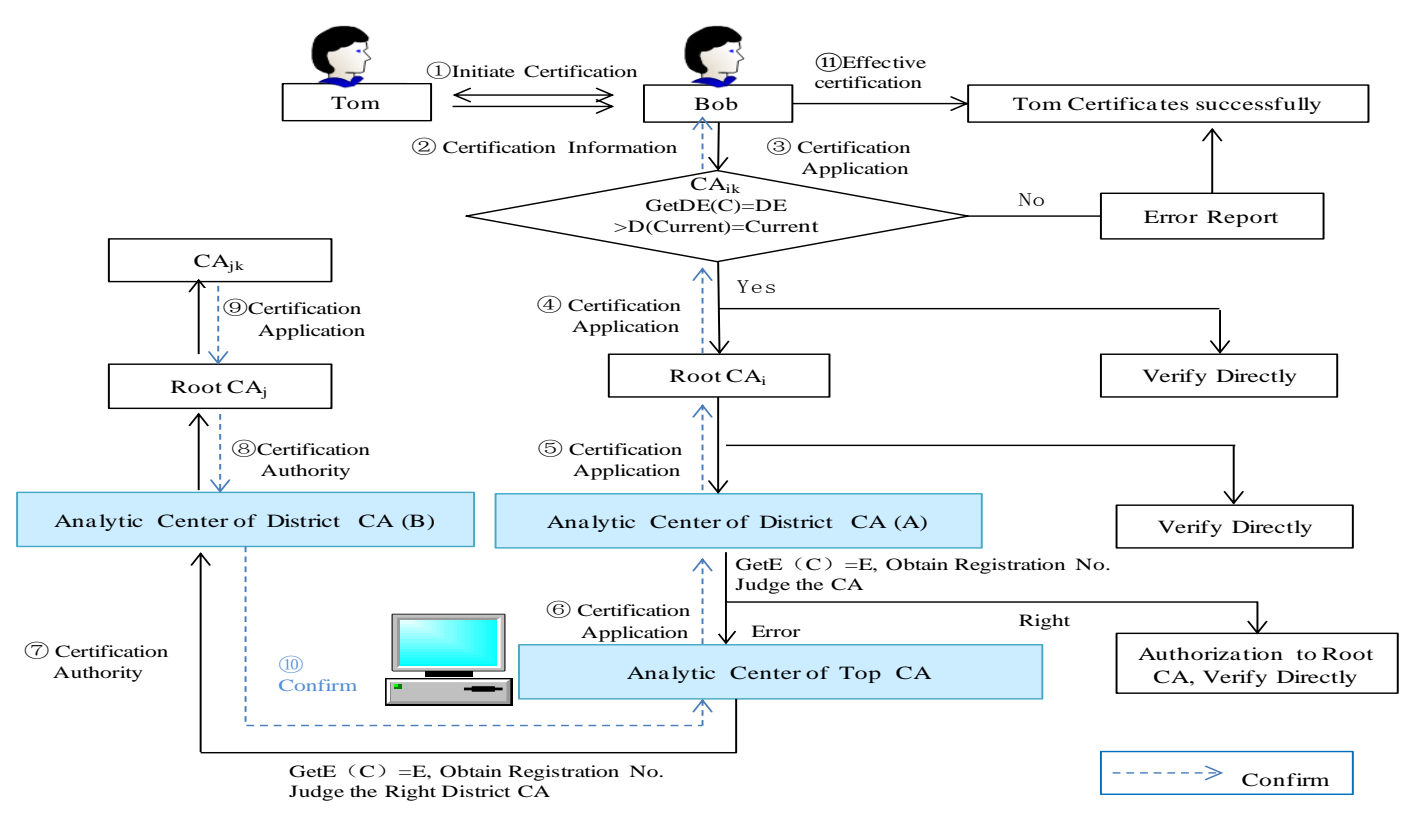

Figure 4. Flow Chart between Different Districts

There are eleven steps for the unified authentication process between different districts. 
(1) Bob needs to verify the authenticity of the public key from Tom's certificate, so they launch authentication to each other.

(2) Bob gets Tom's certificate information.

(3) Bob issues a certification application to his own subordinate $C A_{i k}$.

(4) With information passed by Bob, $C A_{i k}$ judges validity of the certificate. If GetDE $(C)=D E>D($ Current $)=$ Current, which means that the certificate expiration date hasn't achieved yet and the certificate has not expired. Then $C A_{i k}$ continues to determine whether the certificate issued by its own CA, and if so, $C A_{i k}$ will verify directly, if it is not, $C A_{i k}$ will submit application to root CA. If $\operatorname{GetDE}(C)=D E<D($ Current $)=$ Current, it means that the certificate has expired, an error report will be returned to the applicant, Tom.

(5) To determine whether the certificate issued by its own subordinate CA, Root $C A_{i}$ will inspect certificate information. If it is not the correct subordinate CA, Root $C A_{i}$ will apply for authentication from analytic center of district CA (A).

(6) Analytic center of district $\mathrm{CA}(\mathrm{A})$ tests information of digital certificates and obtains a registration number $(G$ et $E(C)=E$ ). Then analytic center determines whether the certificate issued by its own CA, if it is, center will send certificate authority to its root CA, if it is not, center will apply for further authentication from analytic center of top CA.

(7) Analytic center of top CA inspects certificate information, and judges the district of Root $C A_{i}$ by registrationnumber. If the correct district of $C A_{i}$ is $\mathrm{B}$, the center will send certificate authentication to analytic center of district CA (B).

(8) Analytic center of district CA (B) inspects certificate information, and sends certificate authentication to root $C A_{j}$ with registrationnumber.

(9) Root $C A_{j}$ inspects the certificate information and applies for certificate from $C A_{j k}$.

(10) $C A_{j k}$ judges whether the certificate is a valid certificate issued by its own CA. If so, the certificate is confirmed, and confirmation is backtracked. If it is not, the certificate is determined to be invalid, and a confirmation message is backtracked too.

(11) Bob receives the confirmation message, then he judges the validity of confirmation from the unified authentication certificate, finally, Tom will be confirmed if the certificate is valid.

\section{CPNs Modeling}

Coloured Petri Nets (CPNs) is a language for the validation of systems in which concurrency, communication, and synchronization play a major role. The CPN language makes it possible to organize a model as a set of modules. CPN Tools is an industrial-strength computer tool for constructing and analyzing CPN models. It will be possible to verify properties by means of state space methods and model checking and to conduct simulationbased performance analysis with CPN Tools [17]. We will model the scheme according to the flow chart in Figure 3 and Figure 4 by using CPN Tools.

\subsection{Assumptions}

To simplify the model, focus on the verification of this system, we give two assumptions. 
- All communication channels or medium are reliable. So we do not take transport errors, resending messages, and re-receiving messages into consideration when we model the unified authentication system using CPNs.

- We do not consider particular contents and particular formats of all messages, responses and verified results. We focus on the flow charts of the scheme and their reliability and rationality. When we discuss these messages, responses and results, we assume they meet the requirements of the system.

\subsection{Explain to the Declarations and Modules}

The model is shown in Figure5 (Declarations) and Figure6 (The top layer of CPNs model). In this model, the root CA and its subordinate CAs are taken as one local CA.

4.2.1. Declarations: In the declarations, there are several kinds of multi-color sets and variables defined to represent various meanings. They are listed below.

- We declare multi-color sets named STATE_TOR, STATE_TEE, STATE_Local CA, TATE_District CA, STATE_LocalCA2. STATE_Top CA, STATE_DistrictCA2 and TATE_LocalCA3 modeling all of roles, such as, authenticator, authenticatee, local CA $\mathrm{A}$, district $\mathrm{CA}$ analysis center $\mathrm{A}$, local $\mathrm{CA} \mathrm{B}$, top $\mathrm{CA}$ analysis center, district $\mathrm{CA}$ analysis center B and local CA C, in the flow charts defined in Section 3.

- AuthrMessage stands for the set of messages sent during certificating, such as CertificateTee stands for certificate of authenticatee, AuTrue for the authenticated result is true, AuFalse for the authenticated result is false and AuErr for there is an error in certificating.

- $\quad$ EXSTATE_TOR,EXSTATE_TEE,EXSTATE_LCA,EXSTATE_DCA, EXSTATE_ LCA2, EXSTATE_TCA, EXSTATE_DCA2 and EXSTATE_LCA3 are product color sets corresponding with the state color sets defined former and Authr Message.

- AuResult stands for the set of results during certificating, such as TrueCert stands for the certificate of authenticator is true, FlaseCert stands for the certificate of authenticator is False, NonLocalCert for the certificate is not belonged as this local CA and, LocalCANO for that district CA analysis center A checks the certificate and finds the CA's number, NonDistrictCert for the certificate is not belonged as this district CA analysis center, AuError for there is an error in certificate that it could not be found in the database and DistrictNO for that top CA analysis center check the certificate and find the number of district CA analysis center.

- Variables such as stor, stee, slca, sdca, slca2, sdca2, slca3 and stca are declared as type of STATE_TOR, STATE_TEE, STATE_LocalCA, STATE_DistrictCA, STATE_LocalCA2, STATE_DistrictCA2, STATE_LocalCA3, and STATE_TopCA.

- Variables such as aumes_tor, aumes_tee, aumes_lca, aumes_dca, aumes_lca2, aumes_dca2, aumes_lca3, and aumes_tca are all declared as types of AuthrMessage.

- Variable such as auresult is declared as type of AuResult. 


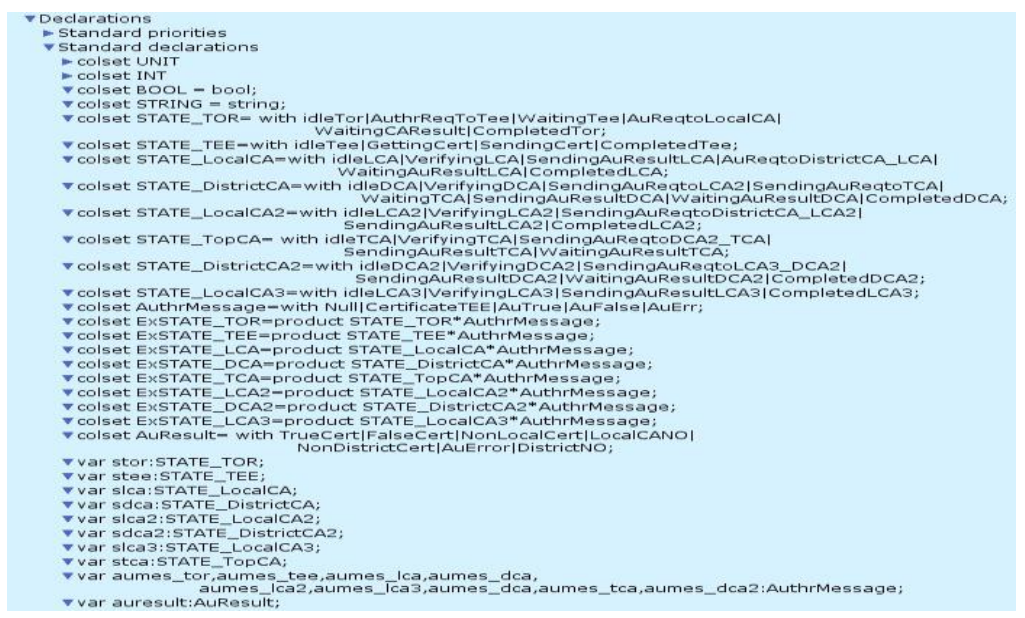

Figure 5. Declaration of the Hierarchical Model

4.2.2. CPN Modules: Because of complexity of the model, we break it into seven smaller pieces (named subpages) and create a hierarchical model. Figure 6 is the top page of the hierarchical model. It contains all roles which include: authenticator, authenticatee, local CA, district $\mathrm{CA}$ and top CA. There are seven substitution transitions that each of them is a subpage between these roles. These substitution transitions are divided by its function such as Auchannel 1 is named as Authenticate Channel 1 (authenticator \& authenticatee) that means it is a communication channel between authenticator and authenticatee. Then, we describe and model these modules by $\mathrm{CPN}$ tools, and final results will be showed in next section.

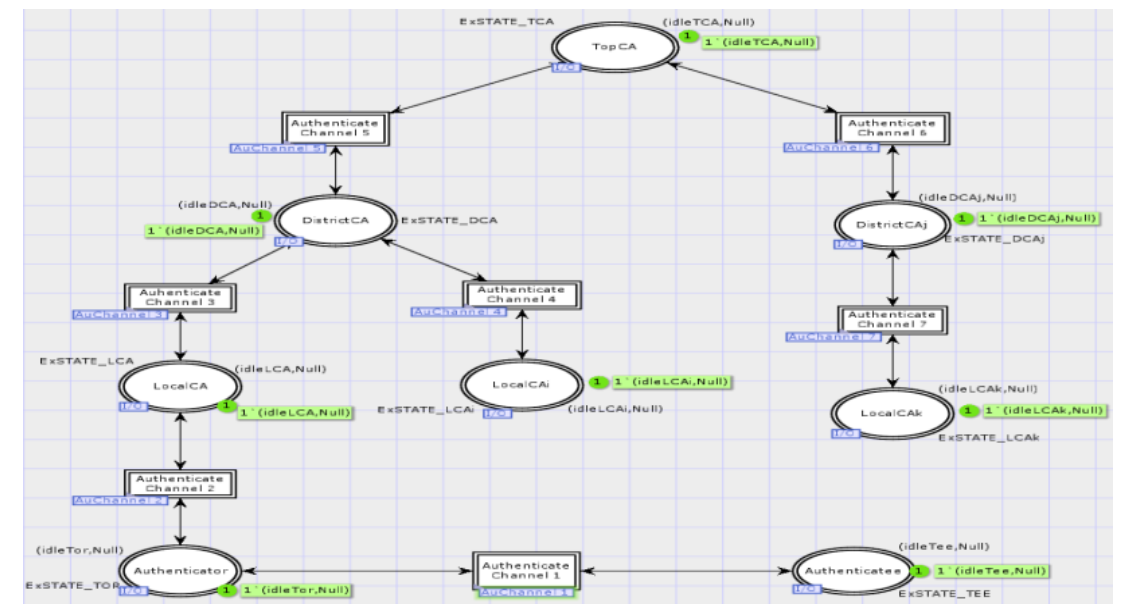

Figure 6. Top Page of the Hierarchical Model

\section{State Space Analysis of the CPN Model}

The state space (SS) report is generated by the CPNs Tools. Part of report is showed in Table 5. It shows that there are 37 nodes and 60 arcs in the state space report and the number of nodes and arcs in SCC Graph is the same as in the SS report. This implies that there is no cycle in this SS report. It means that there no live locks in the model. The table also shows that there is no dead transition instance. So the model has no dead nodes. In the table we find that there are 3 Dead Markings. 
All three dead markings are listed in Table 6, and they have different characteristic. We will discuss the three dead markings in detail.

Table 5. Part of the State Space Report

\begin{tabular}{|c|c|c|c|}
\hline \multirow{4}{*}{ Statistics } & \multirow{3}{*}{ State Space } & Nodes & 37 \\
\cline { 3 - 4 } & & Arcs & 60 \\
\cline { 3 - 4 } & & Secs & 0 \\
\cline { 3 - 4 } & \multirow{3}{*}{ SCC Graph } & Status & Full \\
\cline { 2 - 4 } & & Nodes & 37 \\
\cline { 3 - 4 } & & Arcs & 60 \\
\hline \multirow{3}{*}{ Liveness Properties } & Dead Markings & \multicolumn{2}{|c|}{$3[11,12,13]$} \\
\cline { 3 - 4 } & Dead Transition Instances & \multicolumn{2}{|c|}{ None } \\
\cline { 2 - 3 } & Live Transition Instances & \multicolumn{2}{|c|}{ None } \\
\hline
\end{tabular}

Table 6. Type of Dead Markings

\begin{tabular}{|c|c|l|}
\hline Type & Dead Markings & \multicolumn{1}{c|}{ Characteristic } \\
\hline 1 & 11 & When the certificate of authenticatee is not legal. \\
\hline 2 & 12 & When the certificate of authenticatee is legal. \\
\hline 3 & 13 & $\begin{array}{l}\text { When the verified process of authenticatee's certificate } \\
\text { has any error, such communication error, and so on. }\end{array}$ \\
\hline
\end{tabular}

Through the above table, we can see clearly that there are three final states:

1) State 11, CA certificate is invalid or illegal;

2) State 12, CA certificate is legal;

3) State13, there are communication errors in the verification process, and it is necessary to have an additional verification.

Obviously, these final states are expected by the unified authentication scheme based on analytic mechanism, and there is no infinite loop node and the node will never have an effect, so that all nodes in this certification scheme are reachable. In conclusion, the process of unified certification authentication scheme based on analytic mechanism is feasible.

\section{Conclusions}

In this paper, we present a unified authentication scheme based on analytic mechanism, which is modeled by using CPNs (Colored Petri Nets) tools and we analyze the state space report of the model. Using this unified certification scheme, different CA certificates can be mutually authenticated, and the only one CA certificate issued by the agency can be used for different online payment systems. The contributions of the paper are summarized as below.

- This paper presents a unified authentication scheme based on analytic mechanism, the paper designs registration process for different $\mathrm{CA}$ agencies and extends design-related fields for X.509 V3 certificates, the paper also designs unified certification process within a district as well as between different districts.

- Modeling a unified certification process by CPN Tools. To simplify the model, we give a series of assumptions. Then we take all related entities into consideration for the core places. We declare their states as color sets. Based on these points and workflows, we get the CPN model. 
- Verification of the unified certification authentication process. By analyzing the state space of CPN model, we know that the process has no live lock or dead code and that all three dead markings are desirable terminal states. There is not unexpected marking in this model. So the unified authentication flow of five entities is totally controllable, realizable and feasible.

In the future, we shall revise some assumptions and relax some restrictions. For example we will take unreliable transport medium into account because the real communication medium or Internet is not reliable for payment system.

\section{References}

[1] W. H. Liu, "Construction of CA authentication center", Network Security Technology \& Application, (2014), pp. 213-213.

[2] R. Hunt, "PKI and digital certification infrastructure", Ninth IEEE International Conference on. IEEE, In Networks, (2001) October, pp. 234-239.

[3] M. Qi, "Electronic commerce Security", Higher Education Press, Beijing (2006).

[4] V. Casola, A. Mazzeo, N. Mazzocca and M. Rak, "An innovative policy-based cross certification methodology for public key infrastructures", Public Key Infrastructure, (2005) Springer.

[5] J. Turnbull, "Cross-certification and pki policy networking", Entrust, Inc., (2000) August, pp.1-10.

[6] Y. J. He, "A unified mechanism for cross-certification payment based PKI / CA of the fourth party research", South China University of Technology, (2010).

[7] J. Linn, "Trust models and management in public-key infrastructures", RSA Laboratories, (2000).

[8] P. Alterman, "The US federal PKI and the federal bridge certification authority", Computer Networks, vol. 37 no. 6, (2001), pp. 685-690.

[9] W. T. Polk and N. E. Hastings, "Bridge certification authorities: Connecting b2b public key infrastructures", PKI Forum Meeting Proceedings, (2000) June, pp. 27-29.

[10] M. H. Kharche and M. D. S. Chouhan, "Building Trust in Cloud Using Public Key Infrastructure", International Journal of Advanced Computer Science and Applications, vol. 3, no. 3, (2012).

[11] M. Sudha, "Enhanced Security Framework to Ensure Data Security in Cloud Computing Using Cryptography", Advances in Computer Science and its Applications, vol. 1, no. 1, (2012), pp. 32-37.

[12] Y. W. Sheng, "Research and design on CA in cloud computing", Beijing University of Posts and Telecommunications, (2012).

[13] X. Q. Lai, "The teaching of digital certificates case", Chongqing University of Science and Technology, (2009), pp. $212-213$.

[14] S. A. Brands, "Rethinking public key infrastructures and digital certificates: building in privacy", MIT Press, Cambridge (2000).

[15] D. Solo, R. Housley and W. Ford, "Internet X. 509 public key infrastructure certificate and certificate revocation list (CRL) profile", (2002).

[16] M. R. Thompson, A. Essiari and S. Mudumbai, "Certificate-based authorization policy in a PKI environment", ACM Transactions on Information and System Security (TISSEC), vol. 6, no. 4, (2003), pp. 566-588.

[17] K. Jensen, L. M. Kristensen and L.Wells, "Coloured Petri Nets and CPN Tools for modelling and validation of concurrent systems", International Journal on Software Tools for Technology Transfer, vol. 3-4, (2007).

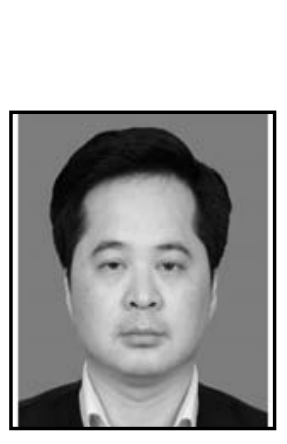

\section{Authors}

Yong $\mathrm{Xu}$, he earned Ph.D. degree of control theory and control engineering from Beijing Institute of Technology, China, in 2000. He now works in South China of University of Technology in Guangzhou City as an associate professor. He published more than 30 research papers in international Journals and Conferences. He published Network Payment and Settle Accounts for Peking University Press. His research areas are in Electronic Commerce, Electronic Payment and Social Network. 


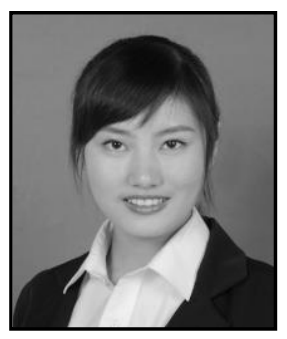

Yejuan Cheng, she obtained her B.S. degree in Mathematics and Applied Mathematics from Anhui University of Finance and Economics, China, in 2013. Now, she is an undergraduate student of Electronic Commerce in South China University of Technology. Her current research field includes Electronic payment and mobile payment.

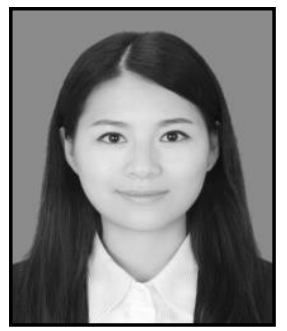

Chang Xiao, she obtained her B.S. degree in Computer Science and Technology from Wuhan University of Science and Technology, China, in 2012. Now, she is an undergraduate student of Electronic Commerce in South China University of Technology. Her current research field includes Electronic payment and mobile payment. 